\title{
Outcomes and Risk Score for Distal Pancreatectomy with Celiac Axis Resection (DP-CAR): An International Multicenter Analysis
}

Sjors Klompmaker, $\mathrm{MD}^{1}$ (D), Niek A. Peters, $\mathrm{MD}^{2,3}$, Jony van Hilst, $\mathrm{MD}^{1}$, Claudio Bassi, $\mathrm{MD}^{4}$, Ugo Boggi, MD, $\mathrm{PhD}^{5}$, Olivier R. Busch, MD, PhD ${ }^{1}$, Willem Niesen, $\mathrm{MD}^{6}$, Thomas M. Van Gulik, MD, PhD ${ }^{1}$, Ammar A. Javed, $\mathrm{MD}^{2}$, Jorg Kleeff, MD, FACS ${ }^{7}$, Manabu Kawai, MD, PhD $^{8}$, Mickael Lesurtel, MD, PhD ${ }^{9}$, Carlo Lombardo, MD $^{5}$, A. James Moser, MD, FACS ${ }^{10}$, Ken-ichi Okada, MD, PhD $^{8}$, Irinel Popescu, MD ${ }^{11}$, Raj Prasad, MD $^{12}$, Roberto Salvia, $\mathrm{MD}^{4}$, Alain Sauvanet, $\mathrm{MD}^{13}$, Christian Sturesson, $\mathrm{MD}^{14}$, Matthew J. Weiss, MD, FACS ${ }^{2}$, Herbert J. Zeh, MD, FACS ${ }^{15}$, Amer H. Zureikat, MD, FACS ${ }^{16}$, Hiroki Yamaue, MD, PhD $^{8}$, Christopher L. Wolfgang, MD, MS, PhD ${ }^{2}$, Melissa E. Hogg, MD, FACS ${ }^{17}$, Marc G. Besselink, MD, PhD ${ }^{1}$, and the E-AHPBA DP-CAR study group

\begin{abstract}
${ }^{1}$ Department of Surgery, Cancer Center Amsterdam, Amsterdam UMC, University of Amsterdam, Amsterdam, The Netherlands; ${ }^{2}$ Department of Surgery, Johns Hopkins Hospital, Baltimore, MD; ${ }^{3}$ Department of Surgery, University of Utrecht Medical Center, Utrecht, The Netherlands; ${ }^{4}$ Department of Surgery, Pancreas Institute University of Verona, Verona, Italy; ${ }^{5}$ Division of General and Transplant Surgery, University of Pisa, Pisa, Italy; ${ }^{6}$ Department of General, Visceral and Transplantation Surgery, Heidelberg University, Heidelberg, Germany; ${ }^{7}$ Department of Visceral, Vascular and Endocrine Surgery, Martin-Luther-University Halle-Wittenberg, Halle, Saale, Germany; ${ }^{8}$ Second Department of Surgery, Wakayama Medical University, Wakayama, Japan; ${ }^{9}$ Department of Surgery and Liver Transplantation, CroixRousse University Hospital, Hospices Civils de Lyon, University of Lyon I, Lyon, France; ${ }^{10}$ The Pancreas and Liver Institute, Beth Israel Deaconess Medical Center, Harvard Medical School, Boston, MA; ${ }^{11}$ Center of General Surgery and Liver Transplant, Fundeni Clinical Institute, Bucharest, Romania; ${ }^{12}$ Department of HPB and Transplant Services, National Health Service, Leeds, UK; ${ }^{13}$ Department of HPB Surgery, Hôpital Beaujon, APHP, University Paris VII, Clichy, France; ${ }^{14}$ Division of Surgery, Department for Clinical Science, Intervention and Technology (CLINTEC), Karolinska Institutet at Karolinska University Hospital, Stockholm, Sweden; ${ }^{15}$ Department of Surgery, University of Texas Southwestern Medical Center, Dallas, TX; ${ }^{16}$ Department of Surgery, University of Pittsburgh Medical Center, Pittsburgh, PA; ${ }^{17}$ Department of Surgery, Northshore University HealthSystem, Chicago, IL
\end{abstract}

Presented at the Pancreas Club, June 2018, Washington D.C., USA. Also presented at the 13th IHPBA World Congress in Geneva, Switzerland.

Collaborators of the E-AHPBA DP-CAR study group are listed in "Acknowledgment".

Electronic supplementary material The online version of this article (https://doi.org/10.1245/s10434-018-07101-0) contains supplementary material, which is available to authorized users.

(C) The Author(s) 2019

First Received: 13 April 2018;

Published Online: 4 January 2019

M. G. Besselink, MD, PhD

e-mail: m.g.besselink@amc.nl

\begin{abstract}
Background. Distal pancreatectomy with celiac axis resection (DP-CAR) is a treatment option for selected patients with pancreatic cancer involving the celiac axis. A recent multicenter European study reported a 90-day mortality rate of $16 \%$, highlighting the importance of patient selection. The authors constructed a risk score to predict 90-day mortality and assessed oncologic outcomes. Methods. This multicenter retrospective cohort study investigated patients undergoing DP-CAR at 20 European centers from 12 countries (model design 2000-2016) and three very-high-volume international centers in the United States and Japan (model validation 2004-2017). The area under receiver operator curve (AUC) and calibration plots were used for validation of the 90-day mortality risk model. Secondary outcomes included resection margin status, adjuvant therapy, and survival.
\end{abstract}


Results. For 191 DP-CAR patients, the 90-day mortality rate was $5.5 \%$ (95 confidence interval $[\mathrm{CI}], 2.2-11 \%$ ) at 5 high-volume ( $\geq 1$ DP-CAR/year) and 18\% (95 CI, 9-30\%) at 18 low-volume DP-CAR centers $(P=0.015)$. A risk score with age, sex, body mass index (BMI), American Society of Anesthesiologists (ASA) score, multivisceral resection, open versus minimally invasive surgery, and low- versus high-volume center performed well in both the design and validation cohorts (AUC, 0.79 vs 0.74; $P=0.642$ ). For 174 patients with pancreatic ductal adenocarcinoma, the R0 resection rate was $60 \%$, neoadjuvant and adjuvant therapies were applied for respectively $69 \%$ and $67 \%$ of the patients, and the median overall survival period was 19 months (95 CI, 15-25 months).

Conclusions. When performed for selected patients at high-volume centers, DP-CAR is associated with acceptable 90-day mortality and overall survival. The authors propose a 90-day mortality risk score to improve patient selection and outcomes, with DP-CAR volume as the dominant predictor.

Distal pancreatectomy with celiac axis resection (DPCAR) may lead to a radical resection for selected patients with locally advanced pancreatic cancer involving the celiac axis. The procedure relies on collateral flow from the superior mesenteric artery via the pancreatic head arcade to the liver and stomach. Some centers perform preoperative embolization of the hepatic and/or left gastric artery ${ }^{1}$ to maximize the formation of collaterals and reduce the risk of ischemia-related complications, but evidence in support of this practice is lacking.

A recent systematic review of 250 patients, ${ }^{2}$ four subsequent single-center studies of 17-80 patients, ${ }^{3-6}$ and a pan-European retrospective multicenter study of 68 patients $^{7}$ all suggested that DP-CAR for pancreatic ductal adenocarcinoma leads to an acceptable overall survival ranging from 17 to 35 months. Despite this survival benefit, the 90-day mortality rate after DP-CAR can be as high as $16-18 \% .^{7-9} \mathrm{~A}$ clinical risk score that evaluates the risk of mortality before surgery could inform shared decision making and improve outcomes through better patient selection. However, such a score is difficult to design for low-volume procedures such as DP-CAR.

A recent study and subsequent international validation (Klompmaker et al., unpublished data) proposed a risk prediction model for major morbidity (including mortality) after standard distal pancreatectomy based on age, sex, American Society of Anesthesiology (ASA) score, body mass index (BMI), multivisceral resection, and open versus minimally invasive approach. ${ }^{10}$ This model reflects the general risk for severe adverse events compared with the risk for specific complications after distal pancreatectomy.
Although DP-CAR is a more extensive procedure, the existing model could serve as a basis for a clinical risk score. It is known that this method of adjustment is superior to designing of a new model with many fewer patients. ${ }^{11}$

This study aimed to create a 90-day mortality risk score for an existing cohort from 20 European DP-CAR centers and to perform an international validation for a combined cohort derived from three very-high-volume centers in the United States and Japan. We hypothesized that the risk score could successfully identify high-risk patients with little expected benefit from DP-CAR.

\section{METHODS}

For this multicenter retrospective study, we used a design cohort, previously collected at 20 European highvolume pancreas surgery centers, ${ }^{7}$ and a retrospective multicenter validation cohort, which included patients from Johns Hopkins Hospital (JHH), Baltimore, MD, USA (2004-2017), the University of Pittsburgh Medical Center (UPMC), Pittsburgh, PA, USA (2007-2017), and the Wakayama Medical University Hospital (WMUH), Wakayama, Japan (2004-2017).

We added and updated the previously published series $^{3,4,12}$ comprising the validation cohort. We used the design cohort to create a 90-day mortality risk score based on the coefficients of a previously validated prediction model for major morbidity, described later in more detail.

The study was designed according to the Strengthening the Reporting of Observational Studies in Epidemiology (STROBE) guidelines for observational studies. ${ }^{13} \mathrm{~A}$ predefined study protocol, including methods and authorship agreements, was distributed among the participating centers. The need for ethical approval was waived by the institutional review board at the Academic Medical Center in Amsterdam.

\section{Exposures and Outcomes}

The DP-CAR procedure was performed in four main variations: (1) standard DP-CAR with resection of the common hepatic, the left gastric, and the splenic arteries, ${ }^{14}$ (2) DP-CAR with superior mesenteric or portal vein resection, (3) DP-CAR with hepatic artery reconstruction (for insufficient flow), and (4) DP-CAR with left gastric artery preservation or bypass reconstruction. ${ }^{12}$ In this study, all variations were treated equally, but the associations between the type of DP-CAR and 90-day mortality were assessed. The primary outcome was 90 -day mortality. The secondary outcomes were major morbidity (ClavienDindo $3 \mathrm{a}-4 \mathrm{~b}$ ), resection margin status, adjuvant therapy, and survival. 


\section{Data Extraction and Definitions}

In both the design and validation cohorts, data were extracted without using patient identifiers. For the validation cohort, we queried all three local databases for patients who underwent DP-CAR for all indications (premalignant or malignant) in adult patients ( $\geq 18$ ). We updated survival on existing cases and added new cases until 1 June 2017.

The preoperative variables included baseline characteristics (age, sex, BMI, Charlson comorbidity index, surgical history), performance status (ASA classification), vascular and/or organ involvement, and tumor etiology. Procedures were considered multivisceral if additional organ resections besides those for pancreas, gallbladder, or spleen were performed.

The annual DP-CAR case volume per center was based on the average number of reported procedures between 1 January 2014 and 31 December 2016. Centers with an annual case volume of one or more were considered highvolume DP-CAR centers, and others were considered lowvolume DP-CAR centers. Due to the lack of variability, annual volume could not be used as a continuous variable.

Postoperative complications were scored and classified according to the Clavien-Dindo classification. ${ }^{15}$ International Study Group on Pancreatic Surgery (ISGPS) definitions were used to classify delayed gastric emptying, post-pancreatectomy hemorrhage, and pancreatic fistulas. ${ }^{16-18}$ Postoperative organ space infections were defined according to the Center for Disease Control and Prevention (CDC) definition. ${ }^{19}$

Centers were asked to categorize resection margins according to the Royal College of Pathologists ${ }^{20}$ definitions as follows: R0 (distance from margin to tumor $\geq 1 \mathrm{~mm}$ ), $\mathrm{R} 1$ (distance from margin to tumor $<1 \mathrm{~mm}$ ), and R2 (macroscopically positive margin). All complications were recorded at the index hospitalization and at subsequent readmissions up to 90 days. Survival was based on the last recorded moment of contact between a patient and a hospital staff member.

\section{Statistical Analysis}

We used predictor estimates from an internationally designed ( $n=1661)$ prediction model for major morbidity (including mortality) after distal pancreatectomy (without arterial resection) based on age, sex, ASA, BMI, multivisceral resection, and open versus minimally invasive approach (Klompmaker et al., unpublished data). We created a risk score by rounding and multiplication of the original model (beta) coefficients.

We tested the model with the design cohort using only baseline (intercept) adjustment and performed validation according to the recommendations by Moons et al. ${ }^{11}$
Validation included addition of new prediction factors based on a univariate screen and forward stepwise selection $(P<0.1)$. Both the intercept adjustment and new predictor coefficients were calculated using the method described by Janssen et al. $^{21}$ We determined the model's ability to identify high- versus low-risk patients (discrimination) by comparing the area under the receiver operating curve $(\mathrm{AUC})^{22}$ in both cohorts using the DeLong test. ${ }^{23} \mathrm{We}$ assessed the accuracy of the risk model predictions per risk quantile using calibration plots. ${ }^{24}$

Categorical variables are reported as counts and proportions and continuous variables as means \pm standard deviations and/or as medians (interquartile ranges) based on normality. To determine statistical significance (alpha 0.05), we used Fisher's exact test for categorical variables and the Mann-Whitney $U$ test for continuous variables. Kaplan-Meier estimation was used to assess overall survival. All confidence intervals are $95 \%$. The data were analyzed using STATA version 15.0 (StataCorp LP, College Station, TX, USA).

\section{RESULTS}

The study investigated 191 patients undergoing DPCAR between 1 January 2000 and 31 June 2017. The design cohort contained 71 patients, and the validation cohort comprised 120 patients. Of these patients, 33 were treated at JHH, 37 at UPMC, and 50 at WMUH. The median follow-up period was 309 days for the design cohort and 447 days for the validation cohort. Overall, 90-day mortality occurred for $18(9.5 \%)$ of the 191 patients. These 18 patients included $11(16 \%)$ in the design cohort and 7 (5.8\%) in the validation cohort (Fig. 1).

The recorded causes of mortality were gastric ischemia $(n=4)$, post-pancreatectomy hemorrhage $(n=4)$, pneumonia $(n=3)$, liver ischemia $(n=2)$, abdominal infection $(n=2)$, sepsis with multi-organ failure $(n=2)$, and progression of residual cancer $(n=1)$. The additional 90-day major morbidity (Clavien-Dindo 3a-4b) rate was $27 \%$, which was comparable in the two cohorts. For 174 patients with pancreatic ductal adenocarcinoma, the R0-resection rate was $60 \%(n=113)$, and the median overall survival period was 19 months (95\% CI 15-25 months). The survival rate did not differ between the patients with $\mathrm{R} 0$ and $\mathrm{R} 1$ resections.

\section{Baseline}

The baseline characteristics are presented in Table 1 . The differences between populations based on the geographic region of origin (Europe vs WMUH vs JHH/ UPMC) are presented in Supplements 1A and 1B. At 


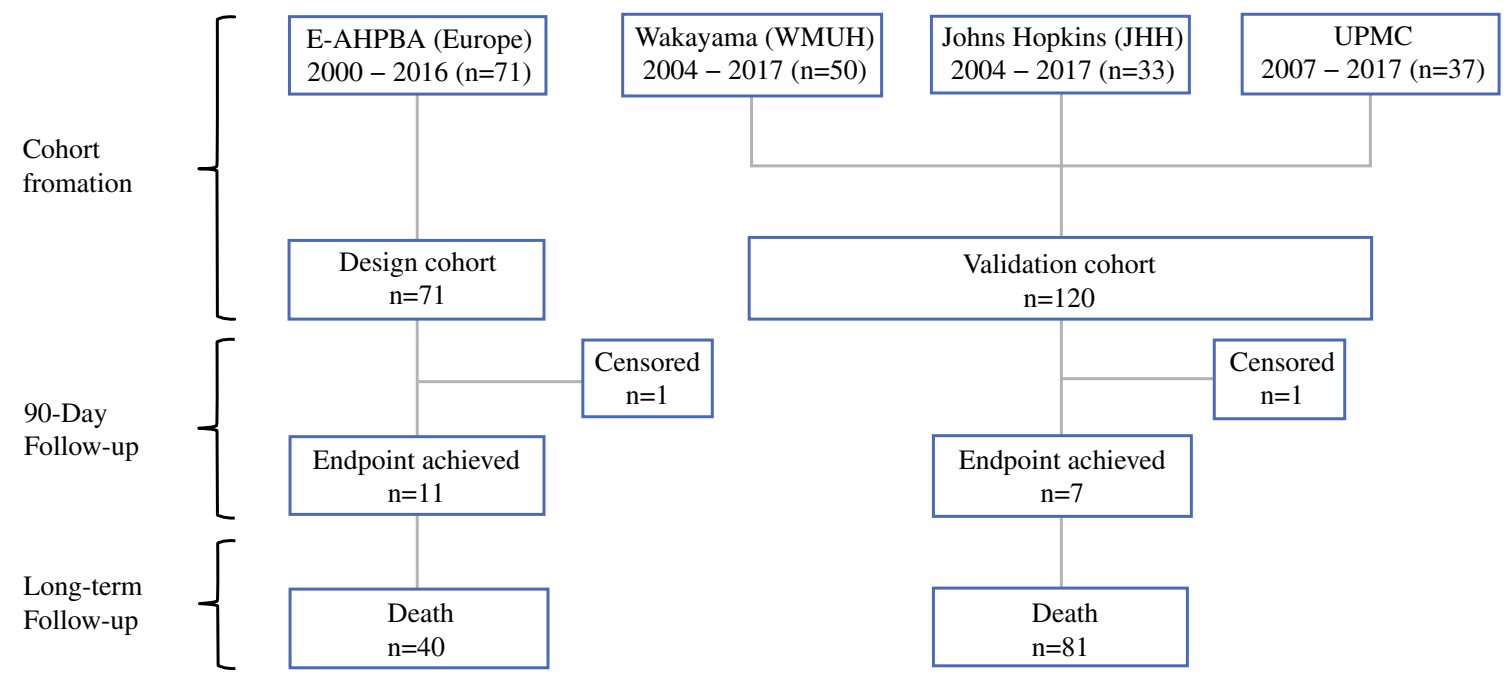

FIG. 1 Study flow chart of data sources and year of inclusion. Two cases were lost to follow-up evaluation within 90 days after surgery.

JHH, Johns Hopkins Hospital; UPMC, University of Pittsburgh E-AHPBA, European-African Hepato-Pancreato-Biliary Association;

TABLE 1 Patient characteristics per cohort

\begin{tabular}{|c|c|c|c|c|c|}
\hline \multirow[t]{2}{*}{ Baseline } & \multicolumn{2}{|l|}{ Design cohort } & \multicolumn{2}{|l|}{ Validation cohort } & \multirow[t]{2}{*}{$P$ Value } \\
\hline & $n=71$ & $\%$ & $n=120$ & $\%$ & \\
\hline Female sex & 34 & 48 & 53 & 44 & 0.654 \\
\hline Median age: years (IQR) & $60(52-67)$ & & $64(58-71)$ & & 0.009 \\
\hline Mean age (years) & $59 \pm 10.6$ & & $63 \pm 10.0$ & & \\
\hline Median BMI: kg/m² (IQR) & $24.0(24-26.3)$ & & $24.4(21.8-27.2)$ & & 0.353 \\
\hline Mean BMI $\left(\mathrm{kg} / \mathrm{m}^{2}\right)$ & $24.3 \pm 3.6$ & & $24.7 \pm 4.2$ & & \\
\hline ASA & & & & & $<0.001$ \\
\hline ASA 1 & 12 & 17 & 2 & 2 & \\
\hline ASA 2 & 53 & 75 & 50 & 42 & \\
\hline ASA 3 or 4 & 6 & 8 & 68 & 57 & \\
\hline Abdominal surgery history $\geq 1$ & 22 & 31 & 53 & 44 & 0.061 \\
\hline Neoadjuvant therapy & & & & & $<0.001$ \\
\hline None & 35 & 49 & 28 & 23 & \\
\hline Chemotherapy & 16 & 23 & 33 & 28 & \\
\hline Radiotherapy & 1 & 1 & 2 & 2 & \\
\hline Both or chemoradiation & 19 & 27 & 57 & 48 & \\
\hline Hepatic artery embolization & 16 & 23 & 46 & 38 & 0.037 \\
\hline Left gastric artery embolization & 6 & 8 & 19 & 16 & 0.185 \\
\hline \multicolumn{6}{|l|}{ Tumor characteristics (pathology) } \\
\hline Ductal adenocarcinoma & 62 & 87 & 113 & 94 & 0.194 \\
\hline Median tumor size: mm (IQR) & $40(34-50)$ & & $33(22-45)$ & & $<0.001$ \\
\hline Mean tumor size (mm) & $47 \pm 29$ & & $34 \pm 18$ & & \\
\hline \multicolumn{6}{|l|}{$\mathrm{AJCC}^{\mathrm{a}}$} \\
\hline $\mathrm{T}$ stage $\geq 3$ & 64 & 90 & 101 & 84 & 0.046 \\
\hline $\mathrm{N}$ stage $>0$ & 46 & 66 & 64 & 53 & 0.168 \\
\hline M stage $>0$ & 1 & 2 & 4 & 3 & 0.655 \\
\hline
\end{tabular}

IQR, interquartile range; BMI, body mass index; ASA, American Society of Anesthesiologists; AJCC, American Joint Committee on Cancer

${ }^{a}$ Based on the 7 th AJCC criteria ${ }^{23}$ 
baseline, the patients from WMUH were less often female (Europe vs WMUH vs JHH/UPMC: $48 \%$ vs $36 \%$ vs $50 \%$, respectively), were older (median 60 vs 68 vs 62 years), and had a lower BMI (mean 24 vs 22 vs 26) than the European and JHH/UPMC-series. Patients at JHH/UPMC were more likely to have an ASA of 3 or 4 ( $8 \%$ vs $19 \%$ vs $84 \%)$ and more likely to receive neoadjuvant therapy (51\% vs $52 \%$ vs $94 \%$ ). Hepatic and/or gastric artery embolization was routinely performed at WMUH $(92 \%)$, and in some cases in Europe (23\%), but never at JHH/UPMC (0\%). The DP-CAR procedure was most often performed for pancreatic ductal adenocarcinoma at JHH/UPMC (88\% vs $87 \%$ vs 97\%), whereas patients in Europe had larger tumors (median 40 vs 30 vs $34 \mathrm{~mm}$ ). Patients in Europe and at WMUH were more likely to have a pathologic American Joint Committee on Cancer (AJCC) T stage 3 cancer or higher ( $90 \%$ vs $98 \%$ vs $74 \%$ ) than patients at JHH/UPMC.

\section{Perioperative and Long-Term Outcomes}

Outcomes are presented in Table 2. Standard DP-CAR (i.e., without venous resection or arterial reconstruction) was most often performed at JHH/UPMC (Europe vs WMUH vs JHH/UPMC: $73 \%$ vs $38 \%$ vs $84 \%$, respectively), and left gastric artery-sparing DP-CAR was often performed at WMUH (46\%), but never in Europe or at JHH/UPMC. The rate of minimally invasive surgery was $18(26 \%)$ at JHH/UPMC and negligible in Europe and at WMUH. The rates for multivisceral resection were comparable between Europe and WMUH (42\% vs $42 \%)$ but lower at JHH/UPMC (30\%).

The 90-day mortality rate was the highest in Europe ( $16 \%$ vs $8 \%$ vs $4 \%)$, and major morbidity was highest at WMUH ( $25 \%$ vs $36 \%$ vs $21 \%$ ). Gastric ischemia was observed in similar proportions (7\% vs $10 \%$ vs $11 \%)$. Strikingly, no liver ischemia was observed at JHH/UPMC ( $19 \%$ vs $56 \%$ vs $0 \%$ ). The JHH/UPMC cohort had the shortest hospital stay (17 vs 21 vs 8 days) but the highest readmission rate (13\% vs $14 \%$ vs $44 \%$ ). The WMUH cohort had the highest rate of adjuvant therapy (63\% vs $80 \%$ vs $74 \%$ ), but the median overall survival, including that after non-pancreatic ductal adenocarcinoma, was highest for the JHH/UPMC cohort (20 vs 16 vs 24 months).

\section{0-Day Mortality Risk Score}

Univariate screening based on baseline characteristics (Supplement 2A) did not show any new predictors for 90-day mortality. Notably, preoperative embolization was not associated with lower rates of 90-day mortality. Screening based on perioperative factors (Supplement 2B) showed that patients without 90-day mortality were significantly more likely to be treated at a high-volume DPCAR center (70\% vs $39 \% ; P=0.015)$ and to have lower operative blood loss (median 500 vs $1050 \mathrm{~mL} ; P=0.021$ ). The observed 90-day mortality rate was $5.5 \%$ (95\% CI $1.5-9.5 \%)$ at 5 high-volume DP-CAR centers (2 US, 1 Japanese, and 2 European centers) and 18\% (95\% CI $7.9-28 \%)$ at 18 low-volume DP-CAR centers $(P=0.015)$. Notably, the type of DP-CAR was not associated with 90-day mortality $(P=0.458)$.

The original risk score performance for distal pancreatectomies is presented in Supplement 3. At model application in both DP-CAR cohorts, the discriminatory power remained stable between the design and validation cohorts (AUC 0.79 vs $0.74 ; P=0.642$; Fig. 2). After baseline adjustment for outcome incidence, calibration was inadequate in the validation cohort (Supplement 4A). We improved model calibration by including low- versus highvolume DP-CAR center as a covariate in the prediction model (Supplement 4B), based on an odds ratio (OR) of 3.71. This translated to 6.5 points on the risk score scale (Table 3).

Final model discrimination, after addition of the volume covariate, remained good (AUC 0.79; 95\% CI, 0.68-0.90). A clinical risk calculator is accessible online at www.panc reascalculator.com.

\section{DISCUSSION}

In this international study of 191 patients treated at 23 centers, DP-CAR was associated with a 90-day mortality rate of $5.5 \%$ in 5 high-volume DP-CAR centers ( $\geq 1$ DPCAR/year) and $18 \%$ in 18 low-volume DP-CAR centers and an additional major morbidity rate of $27 \%$. For 174 patients with pancreatic ductal adenocarcinoma, the R0resection rate was $60 \%$, and the median overall survival period was 19 months.

We created and validated a clinical risk score for 90-day mortality to improve patient selection. The discriminatory power of the risk score was similar between the design and validation cohorts (AUC 0.79 vs $0.74 ; P=0.642$ ). Low annual DP-CAR volume $(<1)$ was the strongest predictor (OR 3.71) of 90-day mortality. The risk score included age, sex, BMI, ASA classification, multivisceral surgery, open versus minimally invasive surgery, and low- versus highvolume DP-CAR center.

In a recent systematic review of DP-CAR, the 90-day mortality rate among 113 patients was $3.5 \% .^{2}$ In addition, two single-center studies reported 90-day mortality rates of $18 \%$ and $17 \%$, respectively. ${ }^{8,9}$ The overall 90 -day mortality rate in the current international multicenter study among 
TABLE 2 Outcomes per cohort

\begin{tabular}{|c|c|c|c|c|c|}
\hline \multirow[t]{2}{*}{ Perioperative } & \multicolumn{2}{|l|}{ Design cohort } & \multicolumn{2}{|l|}{ Validation cohort } & \multirow[t]{2}{*}{$P$ Value } \\
\hline & $n=71$ & $\%$ & $n=120$ & $\%$ & \\
\hline Treated at high-volume DP-CAR centera ${ }^{a}$ & 8 & 11 & 120 & 100 & $<0.001$ \\
\hline Minimally invasive approach & 2 & 3 & 18 & 15 & 0.012 \\
\hline Median operative time: $\min (\mathrm{IQR})$ & $343(248-425)$ & & $350(291-447)$ & & 0.103 \\
\hline Mean operative time (min) & $346 \pm 122$ & & $380 \pm 131$ & & \\
\hline \multicolumn{6}{|l|}{ Additional organs resected ${ }^{\mathrm{b}}$} \\
\hline None & 41 & 58 & 78 & 65 & 0.361 \\
\hline Stomach & 9 & 13 & 8 & 7 & 0.190 \\
\hline Liver & 3 & 4 & 3 & 3 & 0.672 \\
\hline Kidney & 3 & 4 & 3 & 3 & 0.672 \\
\hline Adrenal gland & 17 & 24 & 31 & 26 & 0.864 \\
\hline DP-CAR type & & & & & $<0.001$ \\
\hline Standard DP-CAR & 52 & 73 & 79 & 66 & \\
\hline SMV/portal vein resection ${ }^{c}$ & 10 & 14 & 14 & 12 & \\
\hline Hepatic artery reconstruction & 9 & 13 & 5 & 4 & \\
\hline Left gastric artery preservation/reconstruction & 0 & - & 23 & 19 & \\
\hline Median EBL: mL (IQR) & $560(350-1450)$ & & $560(300-1100)$ & & 0.374 \\
\hline Mean EBL (mL) & $1015 \pm 1145$ & & $996 \pm 1502$ & & \\
\hline Blood transfusion for bleeding $(<72 \mathrm{~h})$ & 22 & 33 & 17 & 14 & 0.005 \\
\hline Residual status overall & & & & & 0.206 \\
\hline R0 (> 1-mm margin) & 38 & 55 & 75 & 63 & \\
\hline $\mathrm{R} 1$ (< 1-mm margin) & 29 & 42 & 38 & 32 & \\
\hline R2 (macroscopically positive) & 2 & 3 & 1 & 1 & \\
\hline \multicolumn{6}{|l|}{ 90-Day outcomes } \\
\hline Mortality & 11 & 16 & 7 & 6 & 0.077 \\
\hline Clavien-Dindo $3 \mathrm{a}-4 \mathrm{~b}$ complication & 18 & 25 & 33 & 28 & 0.866 \\
\hline Post-pancreatectomy hemorrhage $^{\mathrm{d}}$ & 6 & 8 & 9 & 8 & 0.787 \\
\hline Liver ischemia/infarction & 12 & 19 & 28 & 23 & 0.575 \\
\hline Gastric ischemia & 5 & 7 & 13 & 11 & 0.452 \\
\hline Abdominal cavity infection & 4 & 6 & 23 & 19 & 0.016 \\
\hline Pancreatic fistula grade $\mathrm{B} / \mathrm{C}^{\mathrm{d}}$ & 15 & 21 & 27 & 23 & 0.858 \\
\hline Delayed gastric emptying grade $\mathrm{B} / \mathrm{C}^{\mathrm{d}}$ & 11 & 15 & 12 & 10 & 0.495 \\
\hline Reoperation & 10 & 14 & 6 & 5 & 0.018 \\
\hline Median hospital stay: days (IQR) & $17(11-26)$ & & $11(7-21)$ & & 0.005 \\
\hline Mean hospital stay (days) & $20 \pm 14$ & & $18 \pm 21$ & & \\
\hline Unplanned readmission & 9 & 13 & 38 & 32 & 0.005 \\
\hline \multicolumn{6}{|l|}{ Long-term outcomes } \\
\hline Adjuvant treatment & & & & & 0.261 \\
\hline None & 23 & 32 & 26 & 22 & \\
\hline Chemotherapy & 41 & 58 & 72 & 60 & \\
\hline Radiotherapy & 2 & 3 & 3 & 3 & \\
\hline Both or chemoradiation & 2 & 3 & 10 & 8 & \\
\hline Unknown & 3 & 4 & 10 & 8 & \\
\hline Median follow-up: days (IQR) & $309(128-617)$ & & 447 (207-939) & & 0.019 \\
\hline Median overall survival: months $(95 \% \mathrm{CI})$ & $20(10-36)$ & & $21(16-26)$ & & \\
\hline
\end{tabular}

IQR, interquartile range; SMV, superior mesenteric vein; EBL, estimated blood loss; AJCC, American Joint Committee on Cancer; ASA, American Society of Anesthesiologists; CI, confidence interval

${ }^{\mathrm{a}}$ Mean volume of 1 per year between 1 January 2014 and 31 December 2016

${ }^{b}$ Other than celiac axis, gallbladder, pancreas, or spleen

${ }^{\mathrm{c}}$ Excluding side bite

${ }^{\mathrm{d}}$ International Study Group on Pancreatic Surgery (ISGPS) definition ${ }^{16-18}$ 


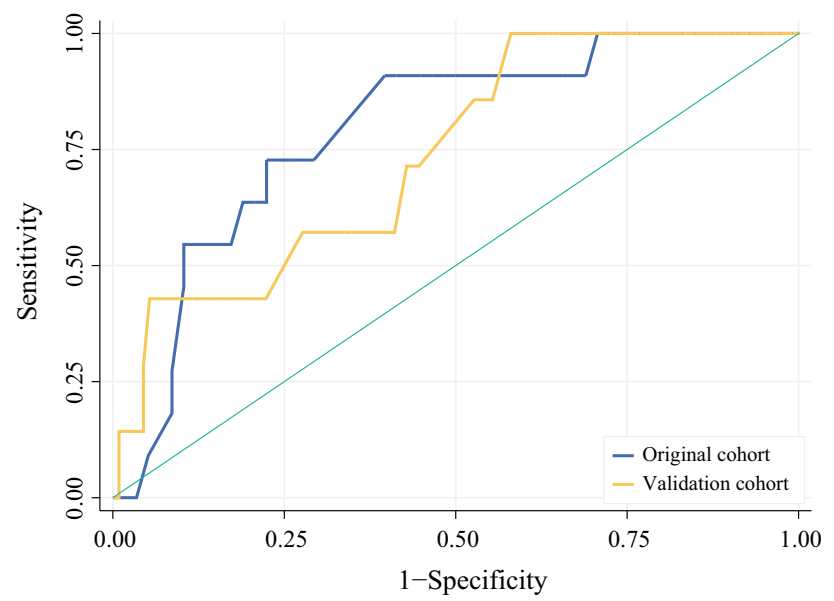

FIG. 2 Discrimination curves for 90-day mortality prediction. Receiver operator curves (ROC) for the 90-day mortality prediction model. The area under the curve (AUC) was 0.79 (95\% confidence interval $[\mathrm{CI}], 0.65-0.93)$ in the design cohort $(n=71)$ and $0.74(95 \%$ CI, 0.56-0.92) in the validation cohort $(n=120)$. The difference is not significant $(P=0.642)$

191 patients was $9.5 \%$. Therefore, it seems likely that publication bias affected prior estimates of the 90-day mortality rate in the systematic review.

On the other hand, our study confirmed previous findings on the volume-outcome relationship in pancreas surgery. For example, a nationwide registry study ${ }^{25}$ found a significantly increased risk of 90-day mortality after pancreatoduodenectomy (OR 2.59; 95\% CI 1.32-5.09) in the lowest- versus the highest-volume centers (cutoff, 40 pancreatoduodenectomies per year). Similar volume-outcome associations for 90-day mortality have been found by others. $^{22,26}$

We noted a few interesting variations in clinical strategies between the three very-high-volume centers in the validation cohort. First, prolonged neoadjuvant treatment is routinely applied (FOLFIRINOX or nab-paclitaxel plus gemcitabine, up to 8 cycles) at JHH and UPMC to assess both tumor biology (aggressiveness) and patient fitness. Second, although some multivisceral resections were necessary during the operation, preoperatively apparent tumor involvement of additional organs is an absolute contraindication for surgery at all three centers. Third, all three centers recognize the need to preserve as much organ perfusion as possible. At WMUH, this is addressed by routine performance of preoperative left gastric and hepatic artery embolization or by preservation or reconstruction of the left gastric artery (middle colic artery bypass). At JHH and UPMC, surgeons refrain from resecting the superior mesenteric artery and perform only partial portal vein resection (side-bites/wedge resection) when involvement is detected intraoperatively. This could explain the low liver ischemia rates and superior survival at UPMC/JHH.
Unfortunately, the subgroups in this study remained too small to study the effect of these strategies on 90-day mortality.

In addition to the clinical strategies described earlier, outcomes such as 90-day mortality after DP-CAR may be improved by using the proposed clinical risk score. Avoiding DP-CAR for high-risk patients would be an obvious step to lowering mortality. Given the limited survival benefit after resection of pancreatic ductal adenocarcinoma, a mortality risk exceeding $10-20 \%$ would not seem justified. Alternatively, the clinical risk score should be used for baseline risk adjustment in future studies comparing specific techniques or center performance.

Despite these practical applications, continuous model reevaluation is important to maintain accuracy. For example, further centralization of pancreatic surgery may shift the definition of "high-volume". Notably, the highvolume centers in this study all performed more than 90 (median 175; interquartile range [IQR], 105-293) pancreatic resections per year.

This study had some limitations. First, despite the large cohorts, it lacked sufficient power to detect less obvious predictors of 90-day mortality. As a result, we may have missed predictors in our clinical risk score. Second, to increase power, all DP-CAR variations were grouped together, whereas outcomes in fact may differ. Future studies should aim to study the differences in long-term outcomes between DP-CAR variations. Third, surgeon and center experience are perhaps the most important determinant of a successful outcome after surgery. Although this is partly reflected in the risk score, surgeons should always consider their individual training and experience. Fourth, we used annual case volume as a surrogate marker for the experience of the surgical team. We acknowledge that this may not capture the full complexity of factors that contribute to improved outcomes at these centers. Moreover, referral patterns may shift and/or external expertise may be acquired. Therefore, the high-volume threshold presented in this study should be used only as a starting point for further discussion on centralization. Fifth, median overall survival times should be interpreted with caution because the median follow-up time was 385 days in both cohorts combined. Sixth, there was a considerable time differential (4-7 years) between the design and validation cohorts, which may have introduced a time-dependent bias. Although this may be the case for the effect of chemo-/ radiotherapy regimens on survival, we previously ruled out any differences over time for morbidity and mortality outcomes in the European population. ${ }^{7}$ Finally, although arguably part of the most important outcomes in oncologic surgery, quality-of-life measures were not available for this retrospective study. 
TABLE 3 Risk score adjustment ${ }^{\mathrm{a}}$

\begin{tabular}{|c|c|c|c|c|}
\hline Risk factor & Original $\beta$ coefficient & Original score & Adjusted $\beta$ coefficient & Adjusted score \\
\hline Age per 10 years $(40-90)$ & 0.11 & 0.5 & - & 0.5 \\
\hline Female with ASA $1-2$ & 0 & 0 & - & 0 \\
\hline Female with ASA 3-4 & 0.52 & 2.5 & - & 2.5 \\
\hline Male (any ASA) & 0.88 & 4.5 & - & 4.5 \\
\hline BMI per 10 points $(20-50)$ & 0.39 & 2 & - & 2 \\
\hline Multivisceral resection $^{\mathrm{a}}$ & 0.42 & 2 & - & 2 \\
\hline Open surgery & 0.49 & 2.5 & - & 2.5 \\
\hline High-volume DP-CAR center ${ }^{\mathrm{b}}$ & - & - & -1.31 & -6.5 \\
\hline Intercept & -2.82 & - & -3.96 & - \\
\hline Total & & $6-23.5$ & & $0-23.5$ \\
\hline
\end{tabular}

Model coefficients were obtained from the original design dataset ${ }^{7}$ to determine the risk score by rounding and multiplication. The intercept was adjusted for the risk of 90-day mortality in the design cohort $(n=71)$; a coefficient for high-volume center was added

ASA, American Society of Anesthesiologists; BMI, body mass index; DP-CAR, distal pancreatectomy with celiac axis resection

${ }^{a}$ Definition: other than celiac axis, gallbladder, pancreas, or spleen

${ }^{\mathrm{b}}$ Annual volume $\geq 1$ DP-CAR

This study has some important practical implications and offers important starting points for further study. First, although the consensus that rare and high-risk procedures such as DP-CAR should be limited to very-high-volume centers is well established, optimal volume thresholds for this procedure have not been determined to date. Even a selective approach (e.g., favorable patient risk factors) to DP-CAR should be avoided at low-volume centers because this would decrease the annual DP-CAR volume even further.

Second, important practice variations between highvolume DP-CAR centers, such as the application of preoperative embolization or chemoradiotherapy, were noted. Although these variations did not modify the effect of volume on 90-day mortality in this study, they may indeed have an impact on long-term outcomes such as survival. These effects are likely to be more evident in a broader study setting such as in the Arterial Study Network. ${ }^{27}$

Third, the actual impact of improved patient selection on outcomes after DP-CAR needs further study.

In conclusion, this study presents the largest international series on DP-CAR to date and includes a validated clinical risk score for 90-day mortality. The main finding is that annual DP-CAR case volume is the most important predictor for 90-day mortality. Future studies should aim at (prospectively) validating the clinical risk score, which was made available online at www.pancreascalculator.com.

ACKNOWLEDGMENT We acknowledge the office of the European-African Hepato-Pancreato-Biliary Association for its support and the Living With Hope (LWH) foundation and Cancer Center Amsterdam for providing travel grants. We are grateful to Sarah L. Gerritsen, Mustapha Adham, M. Teresa Albiol Quer, Frederik Berrevoet, Manuela Cesaretti, Raffaele Dalla Valle, Benjamin Darnis,
Markus K. Diener, Marco Del Chiaro, Thilo H. Hackert, Robert Grützmann, Traian Dumitrascu, Helmut Friess, Seiko Hirono, Arpad Ivanecz, Anastasios Karayiannakis, Giuseppe K. Fusai, Knut J. Labori, Santiago López-Ben, Jean-Yves Mabrut, Motoki Miyazawa, Willem Niesen, Fernando Pardo, Julie Perinel, and Geert Roeyen for data collection/provision and/or local study coordination.

Collaborators Sarah L. Gerritsen (Department of Surgery, Cancer Center Amsterdam, Amsterdam UMC, University of Amsterdam, Amsterdam, the Netherlands); Mustapha Adham (Department of Surgery, Hospices Civils de Lyon (HCL) \& Univeristé Claude Bernard Lyon 1 (UCBL1), Lyon, France); M. Teresa Albiol Quer (Department of Surgery, Hospital Universitari de Girona Dr Josep Trueta, Girona, Spain); Frederik Berrevoet (Department of General and HPB Surgery, Ghent University Hospital, Ghent, Belgium); Manuela Cesaretti (Service de Chirurgie Hépatobiliaire et Pancréatique, Department of HBP Surgery, Hôpital Beaujon, Clichy, France); Raffaele Dalla Valle (Department of Surgery, Hepato-pancreato-biliary Unit, University of Parma, Parma, Italy); Benjamin Darnis (Department of Surgery and Liver Transplantation, Croix Rousse University Hospital, Lyon, France); Markus K. Diener (Department of General, Visceral and Transplantation Surgery University of Heidelberg, Heidelberg, Germany); Marco Del Chiaro (Department of Surgery, University of Colorado, Denver (CO), USA); Thilo H. Hackert (Department of General, Visceral and Transplantation Surgery University of Heidelberg, Heidelberg, Germany); Robert Grützmann (Department of Surgery, University Hospital Erlangen, Erlangen, Germany); Traian Dumitrascu (Center of General Surgery and Liver Transplant, Fundeni Clinical Institute, Bucharest, Romania); Helmut Friess (Department of Surgery, Klinikum rechts der Isar, Technische Universität München, Munich, Germany); Seiko Hirono (Second Department of Surgery, Wakayama Medical University, Wakayama, Japan); Arpad Ivanecz (Department of Abdominal and General Surgery, University Medical centre Maribor, Maribor, Slovenia); Anastasios Karayiannakis (Second Department of Surgery, Democritus University of Thrace, Medical School, Thrace, Greece); Giuseppe K. Fusai (HPB Surgey \& Liver Transplantation Unit Royal Free Hospital, London, UK); Knut J. Labori (Department of Hepato-Pancreato-Biliary Surgery, Oslo University Hospital, Oslo, Norway); Santiago López-Ben (Department of Surgery, Hospital Universitari de Girona Dr Josep Trueta, Girona, Spain); Jean-Yves Mabrut (Department of Surgery and Liver Transplantation, Croix 
Rousse University Hospital, Lyon, France); Motoki Miyazawa (Second Department of Surgery, Wakayama Medical University, Wakayama, Japan); Willem Niesen (Department of General, Visceral and Transplantation Surgery University of Heidelberg, Heidelberg, Germany); Fernando Pardo (Department of HPB \& Transplant Surgery, Clínica Universidad de Navarra, Navarra, Spain); Julie Perinel (Department of Surgery, Hospices Civils de Lyon (HCL) \& Univeristé Claude Bernard Lyon 1 (UCBL1), Lyon, France); Geert Roeyen (Hepatobiliary, Endocrine and Transplantation Surgery, Antwerp University Hospital, Antwerp, Belgium).

OPEN ACCESS This article is distributed under the terms of the Creative Commons Attribution 4.0 International License (http://crea tivecommons.org/licenses/by/4.0/), which permits unrestricted use, distribution, and reproduction in any medium, provided you give appropriate credit to the original author(s) and the source, provide a link to the Creative Commons license, and indicate if changes were made.

\section{REFERENCES}

1. Kondo S, Katoh H, Shimizu T, et al. Preoperative embolization of the common hepatic artery in preparation for radical pancreatectomy for pancreas body cancer. Hepatogastroenterology 2000; 47:1447-9.

2. Klompmaker S, De Rooij T, Korteweg JJ, et al. Systematic review of outcomes after distal pancreatectomy with coeliac axis resection for locally advanced pancreatic cancer. BJS 2016; 103:941-9. https://doi.org/10.1002/bjs.10148.

3. Peters NA, Javed AA, Cameron JL, et al. Modified appleby procedure for pancreatic adenocarcinoma: Does improved neoadjuvant therapy warrant such an aggressive approach? Ann Surg Oncol 2016; 23:3757-64. https://doi.org/10.1245/s10434-0 16-5303-3.

4. Ocuin LM, Miller-Ocuin JL, Novak SM, et al. Robotic and open distal pancreatectomy with celiac axis resection for locally advanced pancreatic body tumors: a single-institutional assessment of perioperative outcomes and survival. $H P B$ 2016; 18:835-42. https://doi.org/10.1016/j.hpb.2016.05.003.

5. Nakamura T, Hirano S, Noji T, et al. Distal Pancreatectomy with en bloc celiac axis resection (modified appleby procedure) for locally advanced pancreatic body cancer: a single-center review of 80 consecutive patients. Ann Surg Oncol 2016; 23(Suppl 5):969-75. https://doi.org/10.1245/s10434-016-5493-8.

6. Sugiura T, Okamura Y, Ito T, Yamamoto Y, Uesaka K. Surgical indications of distal pancreatectomy with celiac axis resection for pancreatic body/tail cancer. World J Surg 2016; 41:258-66. h ttps://doi.org/10.1007/s00268-016-3670-3.

7. Klompmaker S, van Hilst J, Gerritsen SL, et al. Outcomes after distal pancreatectomy with celiac axis resection for pancreatic cancer: a pan-European retrospective cohort study. Ann Surg Oncol 2018; 25:1440-7. https://doi.org/10.1245/s10434-018-639 1-z.

8. Denecke T, Andreou A, Podrabsky P, et al. Distal pancreatectomy with en bloc resection of the celiac trunk for extended pancreatic tumor disease: an interdisciplinary approach. Cardiovasc Intervent Radiol 2011; 34:1058-64. https://doi.org/10.1007/ s00270-010-9997-5.

9. Baumgartner JM, Krasinskas A, Daouadi M, et al. Distal pancreatectomy with en bloc celiac axis resection for locally advanced pancreatic adenocarcinoma following neoadjuvant therapy. J Gastrointest Surg 2012; 16:1152-9. https://doi.org/10. 1007/s11605-012-1839-0.
10. Klompmaker S, van Zoggel D, Watkins AAA, et al. Nationwide evaluation of patient selection for minimally invasive distal pancreatectomy using American College of Surgeons' National Quality Improvement Program. Ann Surg 2017; 266:1055-61. https://doi.org/10.1097/SLA.0000000000001982.

11. Moons KGM, Kengne AP, Grobbee DE, et al. Risk prediction models: II. External validation, model updating, and impact assessment. Heart 2012; 98:691-8. https://doi.org/10.1136/heart jnl-2011-301247.

12. Okada K, Kawai M, Tani M, et al. Preservation of the left gastric artery on the basis of anatomical features in patients undergoing distal pancreatectomy with celiac axis en bloc resection (DPCAR). World J Surg 2014; 38:2980-5. https://doi.org/10.1007/ s00268-014-2702-0.

13. Von Elm E, Altman DG, Egger M, Pocock SJ, Gøtzsche PC, Vandenbroucke JP. The strengthening the reporting of observational studies in epidemiology (STROBE) statement: guidelines for reporting observational studies. Ann Intern Med 2007; 147:573-7. https://doi.org/10.7326/0003-4819-147-8-200710160 -00010 .

14. Nimura $\mathrm{Y}$, Hattory $\mathrm{T}$, Miura K, Nakajima N, Hibi M. Our experience of resection of carcinoma of the body and tail of the pancreas by Appleby's procedure. Operation 1976; 15:885-9.

15. Dindo D, Demartines N, Clavien P-A. Classification of surgical complications: a new proposal with evaluation in a cohort of 6336 patients and results of a survey. Ann Surg 2004; 240:205-13. h ttps://doi.org/10.1097/01.sla.0000133083.54934.ae.

16. Bassi C, Marchegiani G, Dervenis C, et al. The 2016 update of the International Study Group (ISGPS) definition and grading of postoperative pancreatic fistula: 11 years after. Surgery 2017; 161:584-91. https://doi.org/10.1016/j.surg.2016.11.014.

17. Wente MN, Bassi C, Dervenis C, et al. Delayed gastric emptying (DGE) after pancreatic surgery: a suggested definition by the International Study Group of Pancreatic Surgery (ISGPS). Surgery 2007; 142:761-8. https://doi.org/10.1016/j.surg.2007.05. 005 .

18. Wente MN, Veit JA, Bassi C, Dervenis C. Postpancreatectomy hemorrhage (PPH): an International Study Group of Pancreatic Surgery (ISGPS) definition. Surgery 2007; 142:20-5. https://doi. org/10.1016/j.surg.2007.02.001.

19. Mangram AJ, Horan TC, Pearson ML, Silver LC, Jarvis WR. Guideline for prevention of surgical site infection, 1999. Centers for disease control and prevention (CDC) Hospital Infection Control Practices Advisory Committee. Am J Infect Control 1999; 27:97-132; quiz 133-4; discussion 96. https://doi.org/10. 1016/s0196-6553(99)70088-x.

20. The Royal College of Pathologists. Standards and minimum datasets for reporting cancers minimum dataset for the histopathological reporting of pancreatic, ampulla of Vater and bile duct carcinoma. London: The Royal College of Pathologists; 2002.

21. Janssen KJM, Moons KGM, Kalkman CJ, Grobbee DE, Vergouwe Y. Updating methods improved the performance of a clinical prediction model in new patients. J Clin Epidemiol 2008; 61:76-86. https://doi.org/10.1016/j.jclinepi.2007.04.018.

22. Ravaioli M, Pinna AD, Francioni G, et al. A partnership model between high- and low-volume hospitals to improve results in hepatobiliary pancreatic surgery. Ann Surg 2014; 260:871-7. https://doi.org/10.1097/sla.0000000000000975.

23. Compton C, Byrd D, Garcia-Aguilar J, Kurtzman S, Olawaiye A, Washington M. Exocrine and endocrine pancreas. In: Edge S, Byrd D, Compton C, Fritz A, Greene F, Trotti A (eds) AJCC Cancer Staging Manual, 7th edn. Springer, New York, NY, 2010, pp 241-9. https://doi.org/10.1007/978-1-4614-2080-4.

24. Austin PC, Steyerberg EW. Graphical assessment of internal and external calibration of logistic regression models by using loess 
smoothers. Stat Med 2014; 33:517-35. https://doi.org/10.1002/ sim.5941.

25. van der Geest LGMGM, van Rijssen LBB, Molenaar IQQ, et al. Volume-outcome relationships in pancreatoduodenectomy for cancer. $H P B$ 2016; 18:317-24. https://doi.org/10.1016/j.hpb.201 6.01.515.
26. Finks J, Osborne N, Birkmeyer JD. Trends in hospital volume and operative. N Engl J Med 2011; 364:2128-37. https://doi.org/10. 1097/aln.0b013e31822efcdd.

27. Fusai GK, Pereira S, Valente R, Ravikumar R, Lykoudis P. The arterial study network. Published 2015. Retrieved 10 September 2015 at https://www.thearterialstudy.net. 